\title{
A STUDY ON INSTRUCTION FORMATS ON COMPUTER ORGANIZATION AND ARCHITECTURE
}

\author{
Renas Rajab Asaad \\ Department of Computer Science, Nawroz University, Duhok, KRG - Iraq
}

\begin{abstract}
In this article, we'll learn about the concepts of instruction organized in computer organization. On the premise of accessibility of ALU operands sorts of CPU organization is moreover endorsed in this article. When the constructing agent forms an Instruction it changes over the instruction from its memory helpers shape to standard machine language format called the "Instruction organize". Within the preparation of change, the constructing agent must decide the sort of instruction, change over typical names and express documentation to a base/displacement organize, decide the lengths of certain operands, and parse any strict and constants. An instruction arrangement characterizes the format of bits of instruction, in terms of its constituent parts. An instruction arrangement must incorporate an opcode and verifiably or unequivocally, zero or more operands. Each unequivocal operand is referenced utilizing one of tending to modes. Arrange must, certainly or unequivocally, show tending to the mode for each operand. For most instruction sets, more than on instruction used.
\end{abstract}

Keywords: Instruction Format, Computer Architecture, CPU, ALU.

\section{INTRODUCTION}

The physical and coherent structure of computers is regularly portrayed in reference manuals given with the framework. Such manuals clarify the inner development of the CPU, counting the processor registers accessible and their consistent capabilities. They list all hardware-implemented enlightening, indicate their parallel code arrange and give an exact definition of each instruction. A computer will as a rule have an assortment of instruction code groups. It is the work of the control unit inside the CPU to decipher each instruction code and give the vital control capacities required to handle the instruction. The arrange of instruction is as a rule portrayed in a rectangular box symbolizing the bits of the instruction as they show up in memory words or in a control enlist. The bits of the instruction are isolated into bunches called areas. The foremost common areas found in instruction groups[1] .

In order to simplify the matter, the central processing unit is completely synonymous with the processor or the processor, and its method of work lies in the fact that it is in control of all the operations that are performed on the computer, such as logical and arithmetic operations, of course the completion of its task will only be in parallel and with the help of other electronic parts of the Bios, which is the first assistant to the processor, as it coordinates all tasks and transfers data between the central processing and other electronic components, especially the display card, graphics, random access memory, or the so-called Ram.

It should be noted that the CPU performs the processing by applying the firmware commands, which is a set of precise and special code. To understand this more, take the example of operating the computer from the first moment. When the computer is turned on, the processor executes the commands stored in the ROM chip or what we term the BIOS literally and accurately in order to make sure that all the parts of the computer are complete and ready, of course these electronic parts that are checked for readiness during The operation of the computer or two what is called in English POST for Power On Self-Test, some of which are built into the motherboard or motherboard (such as the processor, the north bridge, the south bridge, the ram, the easy input and output, and others), including what is associated with it such as the hard disk, disk reader, mouse Keyboard and others. After the success of the POST process, the wizard delivers the reins to the Windows operating system to complete the operation and thus displays the desktop and the computer is ready to carry out the commands

\section{LITERATURE REVIEW}

Before going into the architecture of the computer, it must be recalled that the first programmable computer (Colossus) was built during the Second World War with the purpose of decoding the codes (that is, it was a specific target). They

Received ZI April 20ZI; Received in revised form 25 April 20ZI; Accepted 25 May 20ZI;

Available online 28June 2021;

dai: 10.46291//CLNTECHvolSiss2pplo-24 
are stored in memory (in other words, if you want to reprogram this computer, you will have to reconnect the cables differently).

Then many multi-purpose programmable computers appeared, and the difference between them and Colossus was the use of Von Neumann's model to build the computer, in which both data and programs were stored in memory, which facilitated the reprogramming process.[1,2]

Computer architecture is the study of how a set of software and physical equipment interact with each other to form a computer system. That is, how the computer system is designed and what technologies are compatible with it 1.[3]

The relationship between CPU and memory:

The data is transferred between the memory and the CPU by printing or reading a copy of the contents of the storage cells from the memory to the appropriate registers in the CPU through a set of data buses, and through the data buses, the CPU is able to extract and read data or programs instructions from the memory by sending a signal Reading from the control unit via the control bus includes sending the address of the required memory cell via the address bus from the CPU to the memory, and by the same to the CPU, the CPU can write data into the memory cells.

\section{ARITHMETIC AND LOGIC UNIT}

It is considered one of the most important components of the CPU as it performs all mathematical, logical and comparison operations. This unit consists of a set of logic circuits.[4]

Among the components of this unit:

1. The Full Adder circuit that adds 3 binary digits and the Half Adder circuit that adds two binary digits.

2. Invertors circuit used to obtain the integral of one or two of the binary number.

3. Accumulator, which is a binary group of cells called the registrar, and it is usually used to keep the results of the executed operations temporarily until they are transferred to the memory or to other units.

4. A group of binary cells that shows the status of the executed operation. This group is called the Status Register or Flag Register, where each cell is allocated to follow a specific case. The arithmetic and logic unit contains an accumulator recorder that is used to store the results of real-time calculations. Arithmetic and logical operations, and the arithmetic and logic unit can receive data through the accumulator, the memory, or the recorder of the CPU, and it receives the control signal from the control unit, where this signal determines the type of operation to be performed in the arithmetic and logic unit.

\section{THE OPERATIONS PERFORMANCE}

A process is one or more of the procedures that are processed and performed by computers, including a processor. The processes themselves are divided into several categories:

Input and output. A number of external devices must be connected to the computer necessarily, for example, keyboard and mouse. They are directly related to the processor and a separate process is assigned to them. It performs data transfer between the CPU and the peripheral devices, and also causes certain actions for the purpose of recording information in memory or its output to external equipment.

System operations are responsible for stopping the operation of the program, regulating data processing, in addition, they are responsible for the stable operation of the computer system.

Writing and uploading operations. Data is transferred between the processor and memory using parcel operations. Speed is provided by simultaneous recording or by uploading groups of commands or data.

In arithmetic, logical terms. This type of operation calculates the values of functions and is responsible for processing numbers, and converting them to various calculus systems.

Transitions. Thanks to shifts, the system speed increases significantly, because they allow you to transfer control to any program team, independently to select the most favorable transition conditions.

Received OG April 20Z1; Received in revised form I0 April 20ZI; Accepted Q6 May 20ZI;

Available online 28June 2021;

dai: 10.46291//CDNTECHval5iss/ppl8-24 
All processes must be running simultaneously, because many programs are running while the system is active. This is done by interleaving the processing of data by the wizard, which allows you to prioritize and implement operations in parallel.[5]

\section{EXECUTION ORDERS}

The command handling is divided into two parts - operation and operation. The operational component shows the entire system what it should be working on at the moment, and the parameter performs the same way, only separately with the processor. Kernels are responsible for executing commands, and actions are executed sequentially. First, there is development, then decoding, actual command execution, memory request, and end result saving.[6]

\section{INSTRUCTION FORMAT}

CPU, short for Central Processing Unit, is the main and important part of a computer, and it is responsible for processing data and carrying out all logic and arithmetic operations, as well as issuing all commands on the computer. What is the working principle of the processing unit, and it's internal components.[7]

Most electronic and mechanical devices contain a central processing unit, which is called in English the Central Processing Unit, which is symbolized by the abbreviation CPU.

It should be noted that the latter are different from one device to another, depending on the nature of the work of each device, so that the CPU in the computer is not the same as in modern cars, for example, and so on. Steps to execute commands within CPU:

1- Loading the instruction: In this process, the program counter directs the memory management unit to the instruction address to be loaded and saved in the Instruction Register, and immediately after that, the counter automatically moves to the address of the following instruction to be ready once the current instruction is executed. This process is called fetch.

2- Instruction analysis: The Instruction Decoding unit analyzes the instruction in the instruction recorder and determines its requirements. This process is called decode.

3- Downloading data: It may be one of the requirements of the instruction to obtain certain data, such as if the instruction is to add two numbers, so the processor needs here to download the two numbers. The memory control circuit directs the processor to the appropriate address to download the data related to the instruction, where it is loaded and saved in one of the recorders.

4- Executing the instruction: The arithmetic and logic unit carries out mathematical or logical operations on the data in the records, as required by the loaded instruction and has been analyzed. This process is called execute

5- The output of writing the result. One of the results of the implementation of the previous instruction may be saving some data in memory, or managing an external device connected to the computer.

After completing the implementation of all operations related to the previous instruction, the department that controls the operations memory directs the processor to the next address in which the next instruction is saved, which has been loaded in the program counter, and the previous steps are repeated until the execution of all the program is completed.[6]

\section{REGISTERS}

They are high-speed storage areas in which data is stored before processing, and include:

MAR (Memory Address Register): Contains the location of the data to be accessed in the memory.

MDR (Memory Data Register): Includes data that is transferred to and from memory.

AC (Accumulator): The place to store the mean results of arithmetic and logical operations.

PC (Program Counter): Titles the next instruction to be executed, and converts it to MDR.

Current Instruction Register: Includes the instruction during its manipulation.[7]

Received UB April 20ZI; Received in revised form I0 April 20ZI; Accepted Q6 May 20Z1;

Available online 28dune 20Z1;

dai: 10.46291//CDNTECHval5iss/ppl8-24 
IBR (Instruction Buffer Register): Instructions that will not be executed directly are placed in it.

\section{Memory unit}

This unit of computer architecture includes RAM (random access memory), which is the primary computer memory (as opposed to a hard disk, which is considered a secondary memory). This memory is fast, easy to access by the CPU, and is divided into header and content sections.

Information on this memory is not permanent and disappears when the power is cut off from the computer, but transferring data from permanent memory (hard disk) to this temporary memory allows the CPU to work more quickly.[8]

\subsection{IMPLEMENTATION PHASE}

After determining the type of operation and determining the addresses of the transactions in the first stage, the second stage begins, where this stage can be summarized in the following steps:

- Transactions are passed from the main memory to the arithmetic and logic unit (reading transactions).

- The Calculation and Logic Unit performs the required operation on transactions.

- The control unit issues the signal for the memory unit to receive the result in the address specified in the instruction.

- Instruction Decoder:

Then in order to implement the process, the instruction code must be analyzed to determine its type and implementation. This task is performed by a special unit inside the control unit called the instruction parser. The length of the instruction code plays a role in determining the composition of the instruction parser as the instruction code determines the number of commands that can be executed if the length of the code is 8 The largest number of orders that can be executed equals 256.

Thus, the instruction parser can be defined as an electronic circuit with a number of entries equal to the length of the instruction's code and a number of outputs equal to the number of command operations that can be executed.[8]

\subsection{Main Memory}

To carry out the work, this work (the program) and the necessary data must be present in the main memory temporarily until the processing process is completed and the results are transferred to the output units. A temporary data recorder called a word recorder and a local control unit, and the memory is connected to the control unit through the address recorder (using address lines) and some control signals to determine the read or write operation.

\subsection{Vector Buses}

It is the way in which data travels between parts of a computer, as all major internal components of the computer are connected with the CPU and memory.

\subsection{A Consists Standard CPU}

Address bus: The address bus holds the data (not the data itself) between the processor and memory.

\section{i. Data bus}

Carries data between the processor, memory unit, and input and output devices.

\section{ii. Control Bus}

Carries control instructions from the CPU (and status signals from other devices) in order to control and coordinate all operations within the computer.

The implementation process takes place through several stages:

Received OG April 20Z1; Received in revised form I0 April 20ZI; Accepted Q6 May 20ZI;

Available online 28June 2021;

doi: 10.46291/ICTNTECHvol5iss/pplo-24 


\section{iii. Fetching instructions and commands}

The first step involves retrieving the information "represented by a number or sequence of numbers" from the program's memory, where the location of the instruction "address" is located in the program's memory by a counter that stores a number specifying the address of the next instruction to be fetched. After the instruction is fetched, the instruction length is increased to contain an address. Next instruction in sequence.[9]

The process of fetching information is a bit slow as the processing unit has to wait to fetch every instruction, but this problem has been largely solved in modern processors by the so-called processor architecture.[10]

\section{INSTRUCTION FORMAT'S STEPS}

At the core of the CPU, there is a set of basic operations that it can perform, called a set of instructions, for example: adding or subtracting two numbers, comparing two numbers, or jumping to different parts of the program. Each basic operation is represented by a mixture of bits which is known as "machine language opcode".[11]

During the execution of the instructions in the machine language program, the processing unit determines the operation to be executed by decoding the previous code, and as mentioned, the instructions consist of the operating code and additional bits that specify the details of the operation (for example the numbers that will be collected in the event of the addition process) .

The actual arithmetic operation of each instruction is performed by an integral logic circuit inside the CPU which is known as ALU. Tom, the processing unit, executes the instructions by fetching it from memory, using ALU to execute the operation, then storing the result on the memory.

Besides arithmetic and logical operations, there are various other instructions such as those for loading data from memory and storing it again, dedicated operations and arithmetic operations on floating point numbers that are performed by the FPU.

When delving into the internal configuration of the CPU, we will encounter some concepts and terms that must be highlighted to fully understand the work of the CPU, which are:

\subsection{Control unit CU}

Theoretically, the CPU can be divided into two units. The first is the control unit (CU) that contains circuits that use electrical signals to direct the entire computer system in order to execute stored program instructions, but the control unit does not execute the program's instructions and instead directs other parts. From the system to do this, the controller connects to both the ALU and the memory.[12]

\subsection{ALU Arithmetic and Logic Unit}

The second half of the processing unit is a digital circuit inside the processor that performs mathematical and logical operations. The inputs to the ALU are the data that is being processed which is called the parameters and the status information from the previous operations and a symbol from the control unit indicating the operation to be performed. Transactions may come from internal CPU records, external memory, or they may be constants generated by the ALU itself.

\subsection{Memory Management Module}

Most high-end computer processors contain a memory management unit that translates logical addresses into physical RAM addresses and provides protection for memory and data transmission systems, especially in microcontrollers.[13]

\subsection{Clock frequency}

Received Q6 April 20ZI; Received in revised form I0 April 20ZI; Accepted Q6 May 20ZI;

Available online 28dune 20Z1;

dai: 10.46291//CDNTECHval5iss/ppl8-24 
Also called CPU frequency, most CPUs are digital synchronous circuits which means they use a clock signal to speed up their serial processes. The clock signal is produced by an external oscillator circuit that generates a constant number of pulses every second in the form of a periodic square wave.[14]

The clock pulse frequency determines the rate at which the CPU executes instructions, so the faster the clock, the more instructions it will execute each second.

Frequency rates have been developed increasingly to improve performance, but they still contain some problems and defects, for example the clock signal is subject to a delay from any other electrical signal, which makes it difficult to maintain the clock signal, which leads to a malfunction of the CPU.

To avoid this problem, modern processing units are equipped with several similar signals around the clock to avoid delaying one signal large enough to cause the unit to malfunction. Another major problem is that the increased clock rates are dissipating a large amount of heat, requiring sophisticated cooling systems to avoid the problems caused by overheating.

\subsection{Performance}

Processor performance or speed depends on many factors such as clock frequency (which is usually given in multiples of hertz) and Hourly Instructions (IPC) which together represent instructions per second (IPS).

CPU performance increases by using multi-core processors that connect two or more processors (called kernels) into a single integrated circuit connecting them.

This means that the processor can handle many asynchronous events, interruptions, and so on, which may affect the CPU when it is overloaded. These cores can be viewed as different floors of a processing plant.[15]

Each floor deals with a different task. Sometimes, these cores will handle the same tasks as their neighboring cores if a single core is not sufficient to handle the information.

Although the CPU is the vital part of the computer, it cannot perform its functions and perform its tasks without other components, so technology is working continuously to develop it and add other sensitive components to its internal structure to increase its efficiency and provide the best possible performance.[16]

\section{CONCLUSION}

In this article, we discussed instruction format and its applications in computer architecture in detail. The central processing unit is the mastermind of the computer, as it is responsible for carrying out all processing operations, including arithmetic and logical operations. This device is connected to the memory, which it uses to process data and instructions.

The control unit and the arithmetic and logic unit make up the central processing unit, which performs two basic tasks: - Executing the program stored in the main memory according to the context of the program's commands and instructions, and modifying the equipment to perform the necessary functions.

The CPU is a set of registers that store data entered into electronic circuits for calculations. The data stored in the registers is just temporary storage before the data is processed and the results are transferred to the main memory, so memory storage is a relatively long-term delayed storage. So that its data and contents are required for processing, while storage on the main processing unit recorders only urgent storage for processing. The recorders are under the direct control of the control unit, which controls data access to the recorders before sending commands to the arithmetic and logical electronic circuits for work. The control unit then monitors the movement and storage of the data in other recorders allocated for the output, and the input recorders can be used to hold the calculations' output.

\section{REFERENCES:}

[1] Lee, G. (1982). Multiple-Address Instructions. In From Hardware to Software (pp. 337-353). Palgrave, London.

[2] Mano, M. M. (2003). Computer system architecture. Prentice-Hall of India.

Received OG April 202I; Received in revised form I0 April 20ZI; Accepted Q6 May 20ZI;

Available online 28June 2021;

dai: 10.46291//CDNTECHval5iss/ppl8-24 
[3] Kuriyama, K., Shintani, Y., Shonai, T., Kamada, E., \& Inoue, K. (1991). U.S. Patent No. 5,075,849. Washington, DC: U.S. Patent and Trademark Office.

[4] Asaad, Renas Rajab. "Review on Deep Learning and Neural Network Implementation for Emotions Recognition." Qubahan Academic Journal 1.1 (2021): 1-4.

[5] Akin, Ö. (2002). Case-based instruction strategies in architecture. Design Studies, 23(4), 407-431.

[6] Asaad, Renas Rajab, and Rasan Ismail Ali. "Back Propagation Neural Network (BPNN) and sigmoid activation function in multi-layer networks." Academic Journal of Nawroz University 8.4 (2019): 216-221.

[7] Asaad, Renas Rajab, and Revink Masoud Abdulhakim. "The Concept of Data Mining and Knowledge Extraction Techniques." Qubahan Academic Journal 1.2 (2021): 17-20.

[8] Asaad, Renas R., and Nisreen L. Abdulnabi. "Using Local Searches Algorithms with Ant Colony Optimization for the Solution of TSP Problems." Academic Journal of Nawroz University 7.3 (2018): 1-6.

[9] Murdocca, M., \& Heuring, V. P. (2000). Principles of computer architecture (pp. 249-251). New Jersey: Prentice Hall.

[10] Almufti, S., R. Asaad, and B. Salim. "Review on Elephant Herding Optimization Algorithm Performance in Solving Optimization Problems." International Journal of Engineering \& Technology 7 (2018): 6109-6114.

[11] Asaad, Renas R. "Güler and Linaro et al Model in an Investigation of the Neuronal Dynamics using noise Comparative Study." Academic Journal of Nawroz University 8.3 (2019): 10-16.

[12] Bacon, D. F., \& Shen, X. (2009). U.S. Patent No. 7,516,306. Washington, DC: U.S. Patent and Trademark Office.

[13] Detmer, R. C. (2001). Introduction to $80 x 86$ assembly language and computer architecture. Jones \& Bartlett Learning.

[14] Asaad, Renas Rajab. An Investigation of the Neuronal Dynamics Under Noisy Rate Functions. Diss. Eastern Mediterranean University (EMU)-Doğu Akdeniz Üniversitesi (DAÜ), 2014.

[15] Tzelnic, P., \& Dunham, S. R. (2002). U.S. Patent No. 6,366,987. Washington, DC: U.S. Patent and Trademark Office.

[16] Lotrich, V. F., Ponton, J. M., Perera, A. S., Deumens, E., Bartlett, R. J., \& Sanders, B. A. (2010). Super instruction architecture of petascale electronic structure software: the story. Molecular Physics, 108(21-23), 3323-3330.

Received U6 April 20ZI; Received in revised form I0 April 20Z1; Accepted Q6 May 20Z1;

Available online 28June 2021;

doi: 10.46291//CTNTECHvol5iss/pplo-24 